\title{
Eye fixations in prediction of recognition and recall
}

\author{
BARBARA TVERSKY \\ Hebrew University, Jerusalem, Israel
}

\begin{abstract}
Eye fixations were recorded at viewing of picture-label stimuli presented under either recall or recognition instructions; both retention tests were administered. Ss performed substantially better on the retention test of which they were informed, indicating differential encoding of the same stimuli in anticipation of test type. There was no correlation between recognition and recall of items, evidence that different information from the encoded stimuli was utilized in performing each test. Encoding strategies had no effect on how Ss regarded the stimuli, but viewing patterns were related to memory performance: More word fixations was associated with better verbal recall, while fewer picture fixations was associated with better recall and with better picture recognition.
\end{abstract}

While it has been amply documented that recognition memory for pictures is remarkably good (Haber, 1970; Nickerson, 1965; Shepard, 1967), little is known about the variables affecting picture memory. Recently, Loftus (1972) has presented evidence that picture recognition improves with the number of eye fixations at viewing. Furthermore, this variable appeared to underlie the effect of exposure time on recognition memory. Loftus suggested that information about the picture is transferred to memory in discrete chunks, each corresponding to an eye fixation. The greater the number of information chunks, the greater the likelihood of correct recognition. Other research (Bahrick \& Boucher, 1968; Frost, 1972; Tversky, 1973) has demonstrated that pictures are encoded into memory differently in expectation of recall than of recognition and that different information from the stimuli is used to pass each test. Ss perform better on that test, recognition or recall, for which they were prepared before viewing the stimuli, and there is virtually no correlation between recognition and recall of an item (Tversky, 1973). Ss expecting recognition access pictorial information more efficiently and those expecting recall access semantic information more efficiently, but both types of information are stored (Frost, 1972). Thus, efficacious encoding anticipates retrieval.

The present experiment extends Loftus's technique to recall of pictures, as well as recognition, in order to explore the relationships between eye fixation patterns (information chunks) and expected memory task

\footnotetext{
*This research was performed while the author held a postdoctoral fellowship from the National Institute of Mental Health at Stanford University. The research was supported by NIMH Grant MH 13950-06 to Stanford University and by the Advanced Projects Research Agency, U.S. Department of Defense, monitored by the U.S. Air Force Office of Scientific Research under Contract F44620-67-C-0099 at the University of Oregon. The author wishes to express her appreciation to Lois Robinson for her very devoted and enthusiastic assistance in data collection and analysis and to Charles Darley for his generous and able assistance in use of the eye camera apparatus. The author is indebted to Arthur W. Melton and to an anonymous reviewer for pointing to an error of interpretation in an earlier version of the paper.
}

(encoding strategies). Eye fixations are recorded during viewing of picture-label stimuli under either recall or recognition instructions; both memory tests follow. Do encoding strategies affect where and how often the $S$ fixates or do encoding strategies affect what information $S$ takes in with each fixation? If eye patterns reflect encoding strategies, then a different fixation pattern is expected under recall than recognition instructions. For instance, Ss anticipating recall might fixate the label more than Ss expecting recognition, who might, instead, fixate details of the picture. On the other hand, if patterns of fixation are governed by aspects of the stimuli rather than by strategic considerations, then eye fixation patterns will not differ under different instructions. Fixation pattern might nevertheless predict memory, but a different relationship might hold between fixations and recognition than between fixations and recall. For instance, number of picture fixations might predict recognition, as in Loftus's results, while number of word fixations might predict recall. This would be consistent with the finding that different information from the same stimulus is accessed for recognition than for recall. Finally, there may be no relationships between fixation patterns and either encoding strategies, on the one hand, or memory task, on the other.

\section{METHOD}

\section{Stimuli}

The stimuli were 30 pairs of slides of line drawings of familiar objects, with their names at the bottom. The drawings were comparable in quality and style to dictionary drawings.

The objects, in order of presentation as well as test, were: books, typewriter, desk, camera, binoculars, pipe, fireplace, television, refrigerator, cake, teapot, iron, umbrella, purse, baggage, barrel, submarine, sailboat, tent, butterfly, fish, kangaroo, tree, skyscraper, windmill, barn, tractor, lawnmower, tricycle, streetcar. For each stimulus, there was another very similar picture with the same name, used as the foil on the recognition test, which differed from the original either by orientation of the object or by internal detail of the object, or by both orientation and detail. There were 10 stimulus pairs of each type; a previous experiment (Tversky, 1973) had shown that these types of visual differences made no difference in either recognition or recall memory. During presentation, stimuli 
Table 1

Mean Percentage Correct Recall and Recognition for Recall and Recognition Instructions $(\mathrm{N}=28)$

\begin{tabular}{lcc} 
& \multicolumn{2}{c}{ Instructions } \\
\cline { 2 - 3 } Test & Recall & Recognition \\
\hline Recall & 59 & 41 \\
Recognition & 66 & 87 \\
\hline
\end{tabular}

were projected for $2 \mathrm{sec}$ each and, during recognition test, pairs of stimuli were projected side by side for $6 \mathrm{sec}$ each.

\section{Subjects}

Ss were recruited from an ad in the local newspaper and were paid for their participation. Of the 33 participants run individually, the data of 5 were eliminated due to technical problems, leaving $28 \mathrm{Ss}$. Of these, half were told that their recognition memory of the pictures would be tested, with each picture paired with a very similar picture of the same objects at test and that their performance would be facilitated by paying close attention to the pictures. The remaining Ss were told that their recall of the object names would be tested and that their performance would be improved by interrelating, associating, or organizing the items on the list. In fact, both recall and recognition were tested. Immediately after viewing the stimuli, Ss were given $3 \mathrm{~min}$ for free written recall of the name of the objects. This was followed by a recognition test, in which pairs of stimuli were presented and S called out "right" or "left," depending on which stimulus he thought he had seen at the presentation.

\section{Apparatus}

Eye movements during presentation of the stimuli were recorded on video tape by means of a wide-angle reflection camera (Mackworth, 1968). The right eye is photographed, and reflected in it is a frame of fluorescent lights surrounding the stimulus. On the video tape, the frame remains stationary, but the pupil moves with each eye fixation so that the number of fixations on each picture can be counted. A black band around each of the vertical fluorescent tubes allowed easy determination of where the $S$ was fixating; when the $S$ fixated the name, the area below the black bands appeared on the center of the pupil, whereas the area above the black bands appeared in the pupil center during a picture fixation. The video tape was scored by a judge who did not know anything about the structure or hypotheses of the experiment. The judge scored the video tape twice independently and then quadruple-checked those few cases which were inconsistent.

\section{RESULTS}

Recognition-informed Ss performed better on the recognition task than recall-informed $S s \quad(p<.001$, median test), and recall-instructed Ss performed better on the recall task ( $<<.05$, median test). Mean percentage correct for each test and instructional condition is shown in Table 1. For each $S$ a phi correlation was calculated to determine the dependency between correct recall and correct recognition of the items. Thirteen Ss showed low $(<+.39)$ positive correlations, 12 showed very low $(<-.19)$ negative, and
3 Ss showed no correlation at all between correct recall and correct recognition of items. The average phi correlation was a very small +.049 , which is not significantly greater than zero $[\mathrm{t}(27)=1.44$ on scores transformed by Fisher's $r$ to $z$ transformation] .

The mean number of eye fixations on pictures and on words for each instructional group is shown in Table 2. Pictures are fixated twice as often as words, irrespective of instructions. In fact, instructions had no significant effect on fixation patterns $[\mathrm{t}(13)=0.97$ for picture fixations and $t(13)=0.92$ for word fixations]. The absolute effect of instructions on fixation pattern was extremely small: Recall-instructed Ss made an average of .13 fixation more on words than recognition-instructed Ss, while recognition-instructed Ss made an average of .09 fixation more on pictures.

Point biserial correlations were computed for each $\mathrm{S}$ between number of picture fixations on an item and recognition of the item and between number of word fixations of an item and recall of that item. The average correlation between picture fixations and recognition was -.07 , which is significantly less than zero $[t(26)=3.07, p<.01]$. The range was from -.30 to +.19 , with 20 Ss with negative correlations and 6 Ss with positive correlations. In the case of word fixations and recall, however, the average correlation was +.13 , which is significantly greater than zero $[\mathrm{t}(27)=3.75, \mathrm{p}<.01]$. Here the range was from -.23 to +.41 , with 21 Ss showing positive correlations and 7 Ss showing negative. There were no effects of instructions on these correlations. Point biserial correlations for each S were also computed between number of picture fixations on an item and recall of that item and between number of word fixations on an item and recognition of the item. The average correlation between picture fixations and recall was -.10 , which is significantly less than zero $[t(27)=3.09, p<.01]$. Scores ranged from -.52 to +.27 , with 20 Ss with negative correlations and 8 Ss with positive correlations. With respect to word fixations and recognition, however, the average correlation was +.06 , which is not significantly different from zero $[\mathrm{t}(26)=1.74, .05<\mathrm{p}<.10]$. In this instance, scores ranged from -.25 to +.41 , with 17 positive and 10 negative scores. Again, there were no effects of instructions on these correlations. Average individual point biserial correlations between total number of eye

Table 2

Mean Number of Word, Picture, and Total Eye Fixations by Instructional Group

\begin{tabular}{lccc}
\hline & \multicolumn{3}{c}{ Instructions } \\
\cline { 2 - 4 } & Recall & Recognition & Total \\
\hline Word & 1.91 & 1.78 & 1.84 \\
Picture & 3.81 & 3.90 & 3.85 \\
Total & 5.72 & 5.68 & 5.70 \\
\hline
\end{tabular}


fixations and each of the memory tasks were very small and not significantly different from zero.

Figure 1 displays the mean percentage correct recall by number of word fixations and instructional groups; Figure 2 displays mean percentage correct recognition by number of picture fixations and instructional groups; and Figure 3 displays mean percentage correct recall by number of picture fixations and instructional groups. In each case, the data from several numbers of fixations are grouped in order to equate as nearly as possible the number of data summarized by each point. The positive relation between word fixations and recall and negative relations between picture fixations and both recognition and recall are evident in these figures.

\section{DISCUSSION}

Instructions preparing Ss either for recognition or for recall of picture-word stimuli led them to perform considerably better on that test for which they had been prepared, replicating previous work (Tversky, 1973). The instructions apparently induced Ss to encode the same stimuli differentially and advantageously for the anticipated memory test. Support for encoding differences between recognition and recall also comes from the work of Tulving and Thomson (1971). These encoding strategies for picture recognition or for free verbal recall were not, however, mediated by selective viewing of the picture as opposed to the word part of the stimuli. Under both sets of instructions, Ss fixated the picture part of the stimulus about twice as many times as the label, perhaps because the label could be

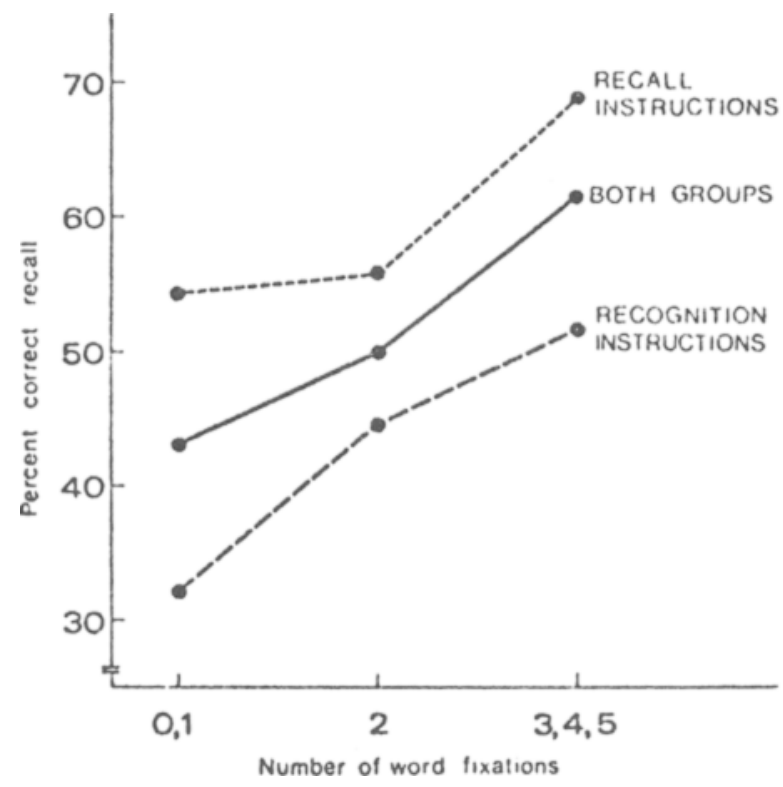

Fig. 1. Mean percentage correct recall as a function of number of word eye fixations at viewing under recall set and recognition set and for both groups together.

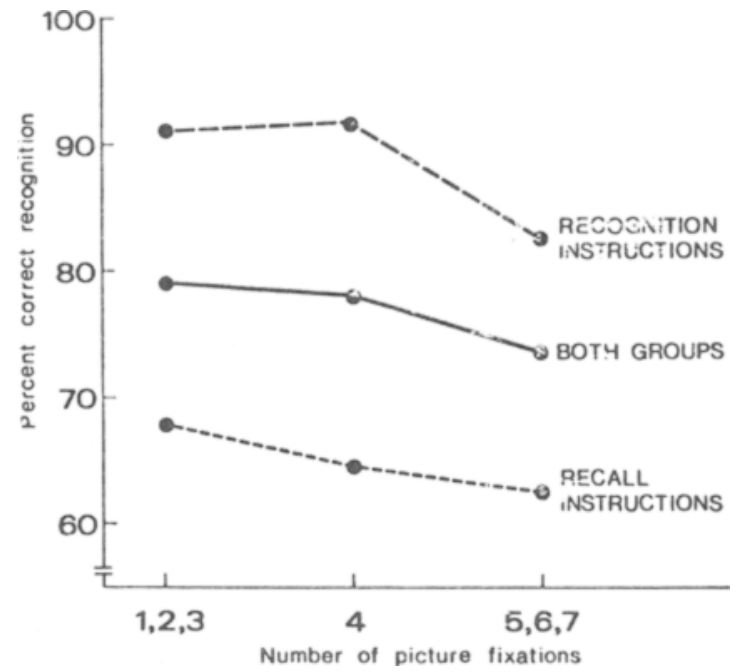

Fig. 2. Mean percentage correct recognition as a function of number of picture eye fixations at viewing under recall set and recognition set and for both groups together.

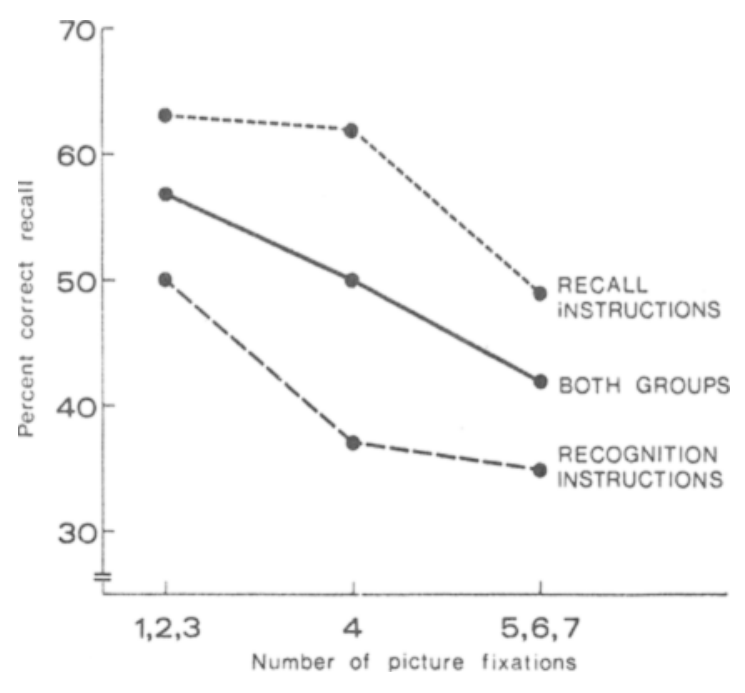

Fig. 3. Mean percentage correct recall as a function of number of picture eye fixations at viewing under recall set and recognition set and for both groups together.

read in the 1.8 fixations typically allotted or else inferred from the picture and perhaps because the picture was more informative (Mackworth \& Morandi, 1967). Studies that have succeeded in demonstrating control of eye fixations by encoding strategies have generally used repeated long trials with the same pictures (Noton \& Stark, 1971) or repeated trials with very simple and structurally similar stimuli (Carpenter \& Just, 1972).

Not only were the stimuli differentially encoded in accordance with the expected memory test but also 
different information was retrieved from the encoded stimuli in performing each of the memory tasks. There was virtually no correlation between correct recognition and correct recall of each item by each $\mathrm{S}$, in accordance with findings of Bahrick and Boucher (1968) and Frost (1972). Successful retrieval in the memory tasks was related to fixation patterns at viewing. Free recall increased with the number of fixations on the label. If a fixation on a visual word is regarded as analogous to verbal rehearsal, this result is consistent with those of Rundus (1971), who found better recall, on the whole, of items given more overt rehearsals. The negative correlations between number of picture fixations and recall and number of picture fixations and recognition are harder to reconcile with the above and with Loftus's (1972) opposite results. Since presentation time was held constant, it is quite likely that, when there were fewer picture fixations, the durations of these fixations were longer, yielding more information per fixation. Such a scanning pattern, where breadth is sacrificed for depth, might well be efficacious in the present recognition task, where success demanded discrimination between pairs of pictures with many features in common. On the other hand, Loftus presented many highly differentiated and easily discriminable stimuli; for such a task cursorily skimming each stimulus may instead be advantageous. In the case of recall, more time per fixations could have been used in interrelating the items. In any case, it is clear that Loftus's finding of a correlation between number of picture fixations and recognition performance cannot be generalized across all types of pictures or all types of memory tasks.

Since the processes in encoding for recognition as opposed to encoding for recall were not reflected in the relative number of picture and word eye movements at viewing, they seem to have had their effect on the manner in which pictorial and verbal information was encoded during fixations. The finding of relationships between both picture and word fixations and recall and between picture fixations and recognition indicates that, in the present tasks, both pictorial and verbal information was utilized for recall, while primarily pictorial information was utilized for recognition. The generality of this finding, however, is limited by the facts that pictures may be verbally encoded and words pictorially encoded (Tversky, 1969) and that the literature on picture recognition and verbal recall contains evidence for pictorial and verbal encoding in each task (Bahrick \& Boucher, 1968; Frost, 1971, 1972; Wyant, Banks, Berger, \& Wright, 1972). Rather than being generally characterized either by type of information selected or by type of encoding modality, it would seem that encoding for recall vs recognition is characterized by differing organizations of the stimuli in anticipation of the retrieval task (Tversky, 1973). Recall is enhanced by the active formation of associations and interrelations among the items, so that retrieval of one item renders easier retrieval of related items, while recognition is enhanced by the integration of the details within an item to facilitate correct discrimination.

\section{REFERENCES}

Bahrick, H. P., \& Boucher, B. Retention of visual and verbal codes of the same stimuli. Journal of Experimental Psychology, 1968, 78, 417-422.

Carpenter, P. A., \& Just, M. A. Semantic control of eye movement in picture scanning during sentence-picture verification. Perception \& Psychophy sics, 1972, 12, 61-64.

Frost, N. Clustering by visual shape in the free recall of pictorial stimuli. Journal of Experimental Psychology, 1971, 88, 409-413.

Frost, N. Encoding and retrieval in visual memory tasks. Journal of Experimental Psychology, 1972, 95, 317-326.

Haber, R. N. How we remember what we see. Scientific American, 1970, 222, 104-112.

Loftus, G. R. Eye fixations and recognition memory for pictures. Cognitive Psychology, 1972, 3, 525-551.

Mackworth, N. H. The wide-angle reflection eye camera for visual choice and pupil size. Perception \& Psychophysics, $1968,3,32-34$.

Mackworth, N. H., \& Morandi, J. A. The gaze selects informative details within pictures. Perception \& Psychophysics, 1967, 2, 547-552.

Nickerson, R. S. Short-term memory for complex meaningful visual configurations: A demonstration of capacity. Canadian Journal of Psychology, 1965, 19, 155-160.

Noton, D., \& Stark, L. Eye movements and visual perception. Scientific American, 1971, 224, 34-43.

Rundus, D. J. Analysis of rehearsal processes in free recall. Journal of Experimental Psychology, 1971, 89, 63-77.

Shepard, R. N. Recognition memory for words, sentences and pictures. Journal of Verbal Learning \& Verbal Behavior, 1967, 6, 156-163.

Tulving, E., \& Thomson, D. M. Retrieval processes in recognition memory: Effects of associative context. Journal of Experimental Psychology, 1971, 87, 116-124.

Tversky, B. Pictorial and verbal encoding in a short-term memory task. Perception \& Psychophysics, 1969, 6, 225-223.

Tversky, B. Encoding processes in recognition and recall. Cognitive Psychology, 1973, 5, 275-287.

Wyant, A., Banks, W. P., Berger, D., \& Wright, P. W. Verbal and pictorial similarity in recognition of pictures. Perception \& Psychophysics, 1972, 12, 151-153.

(Received for publication February 11, 1973; revision accepted September 14, 1973.

Manuscript was lost when returned for revision.) 